\title{
Proposal of the Experimental Promotional Video Model using Image Extension and Contraction, Overlay Techniques: based on augmented Reality and Virtual Museum Concepts
}

\author{
Ga Ram Choi ${ }^{1}$ and Hak Hyun Choi ${ }^{1}$ \\ ${ }^{1}$ Department of Contents Design, Seoul Women's University, 621 Hwarang-ro, \\ Nowon-gu, Seoul, 139-774, Korea \\ grchoi@dclab.kr, choiidea@gmail.com
}

\begin{abstract}
This study is about expressive techniques for promotional videos of cultural-arts organizations, and specifically aims to propose the new video producing model based on the research of Augmented Reality technology and Virtual exhibition. Up to this day, Augmented realty is not popularized for making promotional videos, but it has potential value since this technology can be variously connected with culture contents, specially for exploring exhibitions of museums or art galleries. Therefore, this study aims to examine characteristics of the technology, and find the possibility of combining Information \& communication technology with the culture contents industry specially, for making promotional videos. Then suggest the new experimental design model for making promotional video using the concept, and analyze how the new design model expressive technques make the synergy effect and how the new model is differentiated from the existing promotional video design on the aspects of maximizing user experience and user interaction.
\end{abstract}

Keywords: Culture Contents, Augmented Reality, Virtual Exhibition, 3D Imaging technique, Motion Tracking

\section{Introduction}

The experimental video is edited asing video sources taken at National Museum of Korea, South Korea for the study The yideo, which is based on AR and imitating a Virtual museum with mobile device, attracts people by giving them differentiated experience such as seeing 3D images of objects or getting information via mobile screen. That also makes users get information effectively by feeling like they are actually in the museum and exploring it. On the other words, this study suggests a brand new, specialized study model, not like simple mixture of images and video clips. Then take a look at the main expected effects and further direction of improvement based on an analysis result of the new expressive techniques. Even though the importance of culture contents is becoming critical and ICT is applied on diverse of industrles, there is no notable development in the promotional video field. Promotional videos are still relying on simple images or video clips. Considering tons of promotional videos hit the market every day, and companies are focusing on making promotional videos hopping to reach to consumers via Web, Mobile, TV, etc, flat promotional videos cannot attract users, and that is far away from the recent trend. Therefore, this study aims to examine $\mathrm{AR}$, one of the uprising ICT, see how we can use it for promotion culture contents, and suggest the study model for cultural-art organizations. 


\section{Related Works}

\subsection{Virtual Exhibition}

Lots of museums have been started building cyber space to give users special experience. For example, the virtual exhibition service lets people visit the museum whenever they want, mostly for free by simply connecting on the internet. They don't have to go to the actual place or spend a lot of time for that. According to the research by Gomes and Cláudio, virtual exhibition is valuable for other reasons. For example, virtual visits are valuable to extend the period of an exhibition; to allow visitors to observe objects of art that are seldom exposed or temporarily not available to exposition; to provide additional information or experience to onsite visitors etc. [1]. For example At the Provincial Archaeological Museum of Ename, close to the Ename archeological site, referred above, this was achieved using QuickTimeVR technology [8] to show the evolution of the entire village over the past ten centuries considering 12 distinct periods in time [2]. There are few sample steps for buid ding the Virtual exhibition. Designing the application, Standardization of the Scene Geometry Selection of Surfaces and Placement of Objects are those. Here is a figure showing the contponents of the Application.

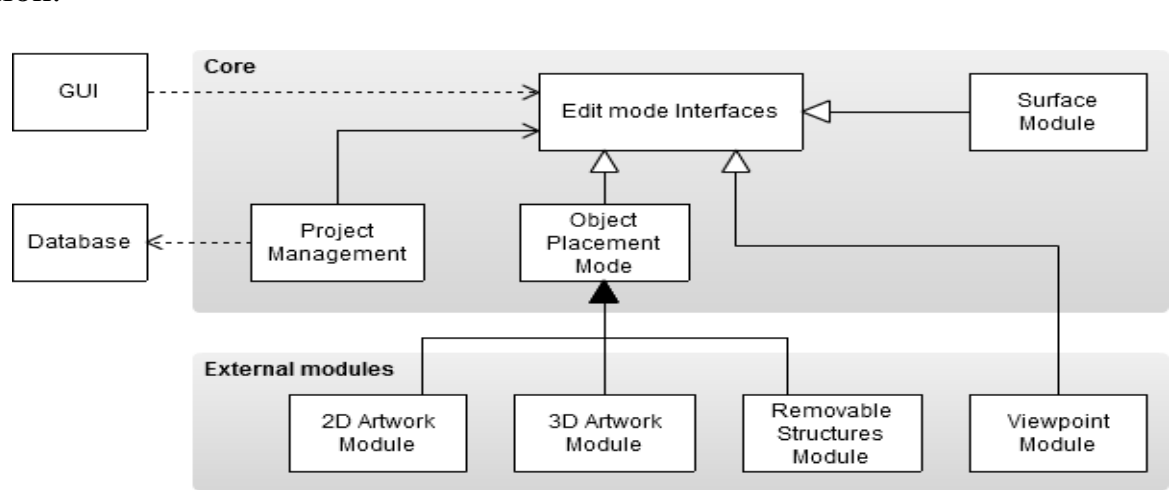

Figure 1. The Components of the Application [1]

On the placement of objects stage, the producer place 2Dimentioanl, 3Dimentioanl, or video as needed. Each type of objects is implemented by a corresponding module which reflects its specificities. As the research by Gomes and Cláudio said, 2D artworks are stored as images in the database, along with additional information about the piece.3D artworks are stored as X3D models.
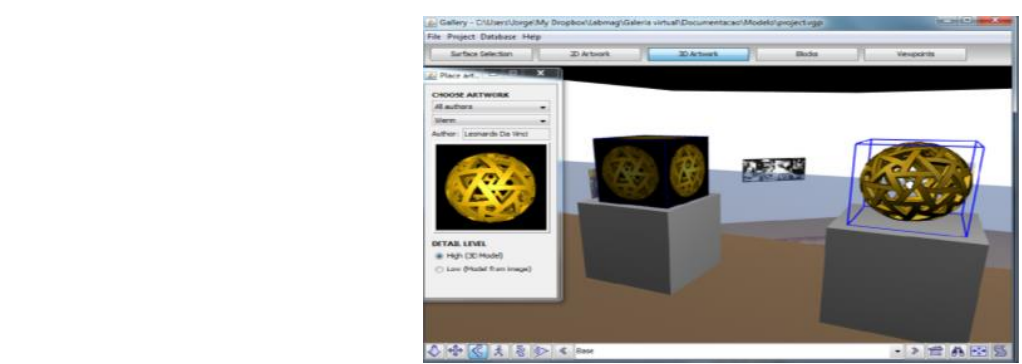

Figure 2. Placement of 3D Objects for the Virtual Exhibition [1]

Here is another example of virtual exhibition. Valentino Garavani Virtual Museumstarted its virtual museum service in 2011. The virtual museum is a desktop application, which can 
be downloaded through internet. This virual exhibition enables people to experience 5 decades of fashion history. This virtual museum serves a differentiated service by letting them explore 3Dimentional museum just like they are actually in the place. User can move forward by clicking and stop by right clicking. User also can move to up, down, sidespace by clicking each side and enter to another exhibition room by calling up the site map and clicking one of the exhibition rooms.

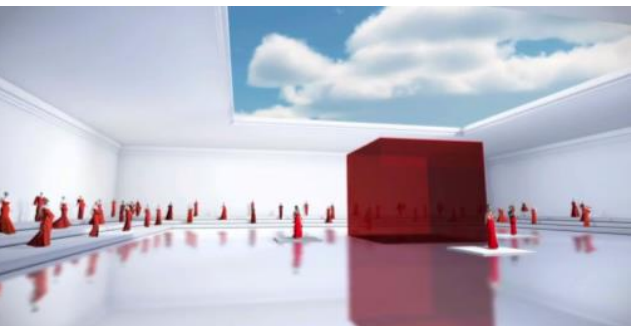

\section{Figure 3. The Valentino Garavani Virtuâl Museum}

\subsection{Image Mapping}

Blaise Agüera y Arcas, a software architecture and designer, held a presentation at TED in 2010 about the new Imaging and video mapping service using augmented reality. He showed some results of the experiments using Google Bing Maps service, which was not about finding directions or navigating. The system shows 3D limages inside and outside of buildings, and enables to get into the building or see cloud sourced pictures or videos of the place.

2.2.1. Zoom In / Zoom Out: Using this experimental model, user can search a certain place and fly down to the map to get closer view of it They can see panoramic images of the place and proceed forward or backward, which has a function of geometry and 3D understanding behind it. In other word, user can Zoon into the map to get to the front side of the building and see 3D-processed images of inside. Also, the map contains specific pictures of inside which was taken ung a small backpack camera.

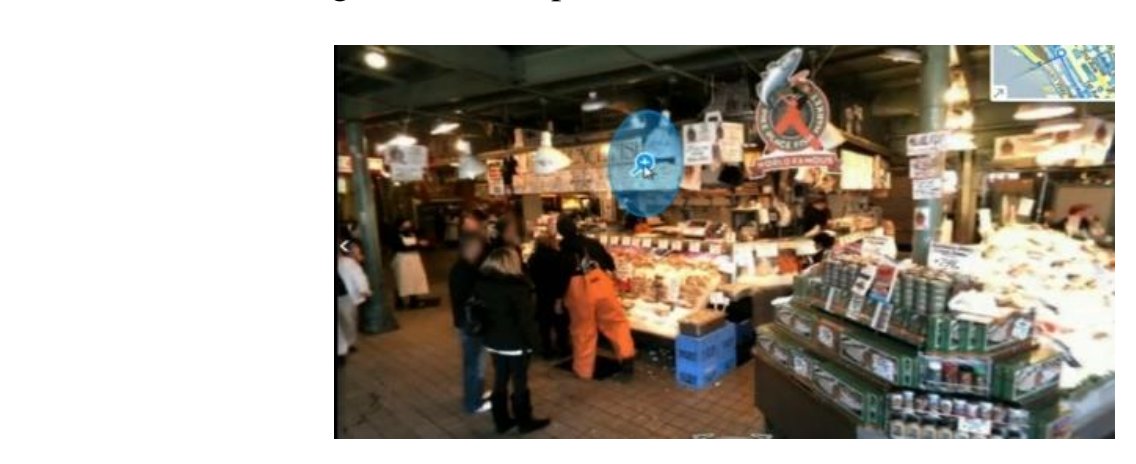

Figure 4. The 3D Processed Image Experiment

2.2.2. Image Mapping Using AR: Blaise Agüera y Arcas introduces the image mapping service using Augmented Reality. When users get to the certain spot in the cyber map, it shows pictures of the spot uploaded by other people. This cloud-sourced (a compound formed from the words 'Cloud' and 'Outsourcing') image mapping technique enables socalled 'Time Travel', which displays different pictures of the spot taken in different time, or whether conditions. The concept of time travel makes users participate in making contents. 
They produce the contents by uploading pictures of videos they took on the spot, and consume by seeing what others did. This experience gives users a sense of satisfaction and they can be active users. This image mapping technology is a foundation of the following AR video mapping technique.

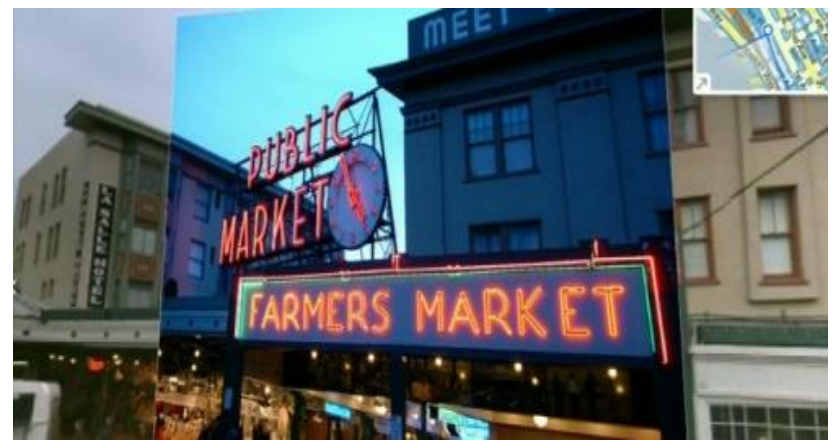

Figure 5. The Image Mapping Experiment

2.2.3. Video Mapping using AR: On the top of Image mapping, mapping videos on the map can be realized using Augmented Reality. Users get to one spot and the real time or uploaded videos are shown on the screen. That makes users feel like they are actually on the spot, and they can also produce video contents as they can do on the image mapping stage. Video mapping can be used for Tele-presence, which means users get close to the spot via internet or mobile applications and experience a sense of realism. This service gives users special experience that they are watching the real time video at home using the cyber map, and being a producer plus consumer.

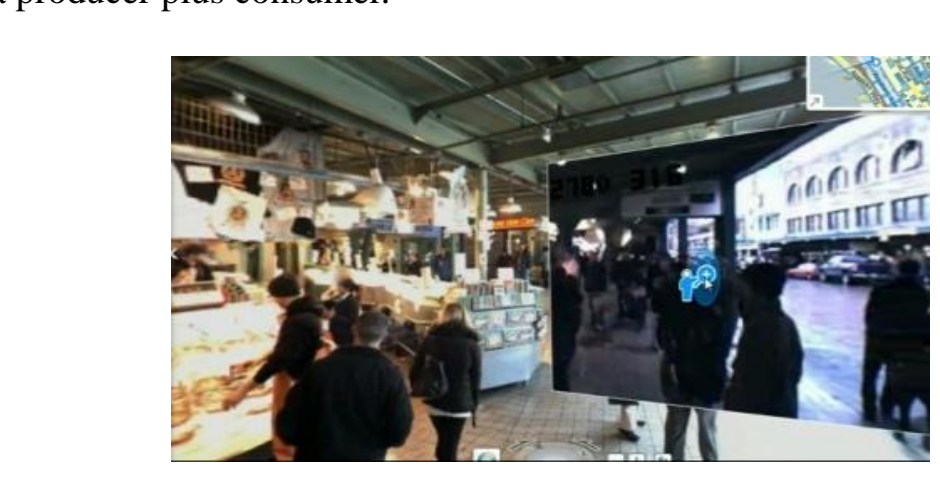

Figure 6. The Video Mapping Experiment

\subsection{Analysis of Augmented Reality}

2.3. Augmented Reality: Augmented Reality (AR) is the technology showing virtual images on the top of real image or background, which is mainly focusing on increasing the interaction between human and computer using 3D processed graphic [3]. Augmented reality is a variation of Virtual reality that allows the user to see the real world with virtual objects imposed upon or composited with the real world [4]. Augmented Reality is called Mixed Reality since AR is adding virtual information on the real environment, which is different from virtual reality. Augmented Reality technology introduced in Communications of the $\mathrm{ACM}$ 의 special issue (July 1993) for the first time [5]. 


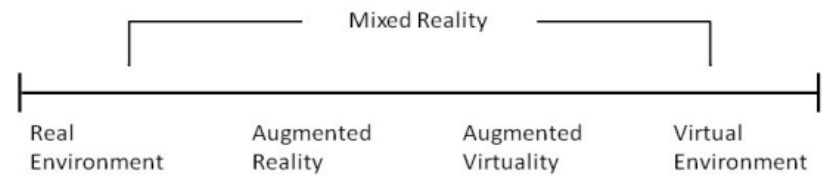

Figure 7. Milgram's Reality-virtuality Continuum [6]

In 1994, Paul Milgram and Fumio Kishino defined 'reality-virtuality continuum'. As shown on Figure 7, Augmented Virtualityis located between Real Environment and Virtual Environment.

\subsubsection{Principles and Application of Augmented Reality}

Table 1. Example of Augmented Reality Approach [5]

\begin{tabular}{|c|c|c|}
\hline Augment: & Approach & Applications \\
\hline Users & $\begin{array}{l}\text { Wear devices } \\
\text { on the body }\end{array}$ & $\begin{array}{l}\text { VR helmets } \\
\text { Goggles } \\
\text { Data gloyes }\end{array}$ \\
\hline Physical objects & $\begin{array}{l}\text { Imbed devices } \\
\text { within objects }\end{array}$ & $\begin{array}{ll}\text { Intellig nt bricks } & \text { Edtcation } \\
\text { Sensors, teceptors } & \text { ffic lacilities } \\
\text { GPS electronic paper } & \text { Postioning }\end{array}$ \\
\hline $\begin{array}{l}\text { Environment } \\
\text { surrounding objects } \\
\text { and users }\end{array}$ & $\begin{array}{l}\text { Project images } \\
\text { record remotely }\end{array}$ & $\begin{array}{ll}\text { Wdeo vameras, Scanter } & \text { Office work } \\
\text { Braphics tablets } & \text { Film-making } \\
\text { B r code readers } & \text { Construction } \\
\text { Video Proje to s } & \text { Architecture }\end{array}$ \\
\hline
\end{tabular}

Table 1 above is the approach to 3 elements of Augmented Reality, which are users, Physical objects, Environment surrounding objects and users. Each approach has pros and cons and can be choose as the purpose of usage. The important point is clearly defining how to make objects and humans interact identifying additional computing problems and technically developing right solutions [5]. There are some requisites for Augmented Reality such as the GPS system, the gravitational sensor (or the gyroscope sensor), the positioning system, etc. The positioning system gets angle, location information and sends it to Augmented Reality applications. Augmented Reality uses those elements to take information, processes it, and prints out the output, which is the final images of information mapping, via Smartphone or Tablet PC

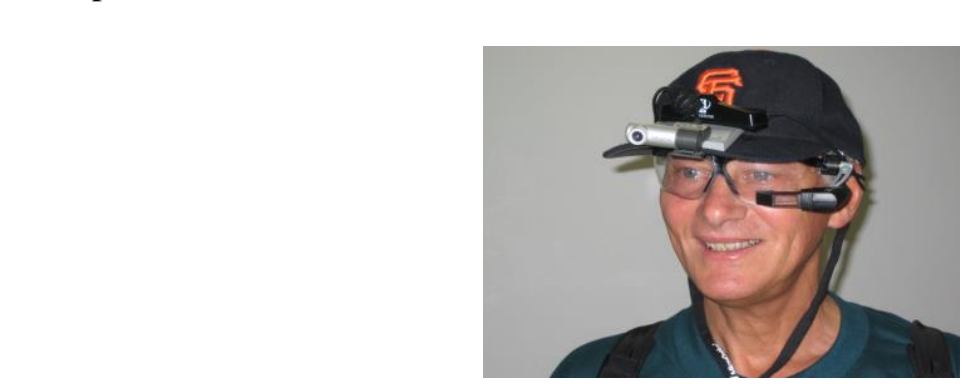

Figure 8. An Example of a Wearable Augmented Reality Display [9]

Augmented Reality is useful for visualizing objects. For example, a research of European Computer-Industry Research Center (ECRC) introduces the experimental AR showing names of a component of an engine when user is pointing one of the components. Seen in Figure 9 below, user points exhaust manifold and the annotation 'exhaust manifold' shows up on the 
screen. This annotation and visualization can be used for many fields. Specially, it is quite useful in a cultural-art industry since museums and art galleries are mostly dealing with physical objects.

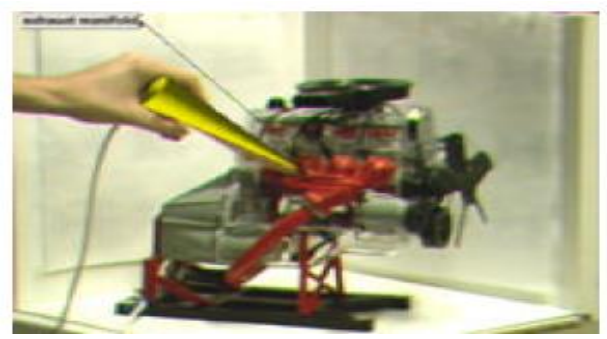

Figure 9. ECRC's Annotation Research using AR

For the more, Augmented Reality technology enables tracking moving objects A system senses the objects and shows information of them. According to a research held by Columbia University [4], a guy is wearing track-able devices on him and the computer tracks his location. Then information label moves along the guy moves and keeps showing information. Figure 10 shows the experiment.

\section{Figure10. Augmented Reality Tracking [4]}

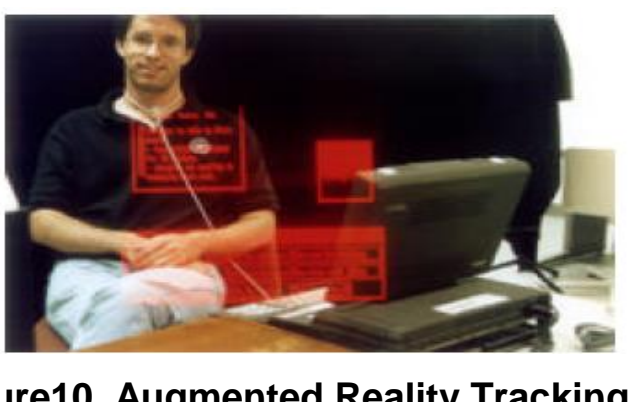

2.3.3. Mobile Angmented Reality: Here is a promotional video of UNIVERSAL Pictures. They used 66,000 AR technologies using mobile applications for $100^{\text {th }}$ yearsanniversity. AR technology was used to lea asers experience their products or contents with mobile applications.

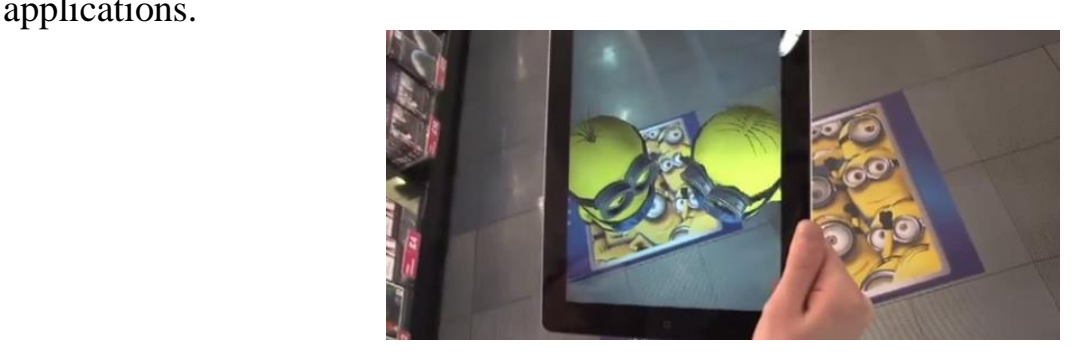

Figure 11. Universal Pictures Augmented Reality DVD - Blu Ray Covers

In the first picture above, by focusing the AR applications on a poster on the floor, 3D movement of the characters is showing up on the screen and showing dancing performance. Users also experience AR at the photo wall. When they stand beside characters and focus the AR applications on the photo wall, the characters on the screen dances with users. 


\subsection{Analysis of Existing Promotional Videos}

Table 2. Analysis of Existing Promotional Videos

\begin{tabular}{|c|c|c|}
\hline & Promotional video A & Promotional video B \\
\hline Sample Images & & \\
\hline Analysis & $\begin{array}{l}\text { Promotional video A, promoting } \\
\text { MUSEUM KAMPA, has a running time } \\
\text { of 02:09. The video starts with pieces of } \\
\text { Google map images of the location fly } \\
\text { into the screen from the right side, and } \\
\text { text shows on the empty space. The video } \\
\text { processes using typography to deliver } \\
\text { information. The video delivers } \\
\text { information by showing video scenes of } \\
\text { the museum, people, and objects along } \\
\text { with appropriately arranged text. At the } \\
\text { end of the video, they shows internet } \\
\text { browser to introduce an art project held } \\
\text { wit Google.. } \\
\text { The Mouse clicking effect and browser } \\
\text { screen get people excited. } \\
\text { The opening scene can be realized using } \\
\text { Premiere Motion effect, wbich is also } \\
\text { used for making an experimental video of } \\
\text { this study. }\end{array}$ & $\begin{array}{l}\text { Video B is promoting the virtual } \\
\text { museum of Valentino Garavani, The } \\
\text { Valentino Garavani Virtual Museum. } \\
\text { The video runs for 01:55, and entire } \\
\text { video consists of the virtual 3D } \\
\text { environment. } \\
\text { As the video proceeds followng the } \\
\text { virtual designed space and contents, } \\
\text { users sees } 2 \text { dimentional } 3 \text { dimentional } \\
\text { images and videos. } \\
\text { Without using audio narration or long } \\
\text { text, information is delivered with } \\
\text { virtual images and simple text. } \\
\text { On the other hand, since this video } \\
\text { show all the information visually, it's } \\
\text { not really clear what they are really } \\
\text { saying. Also the whole virtual scenes } \\
\text { are not enough to interact with users } \\
\text { and get their emotional sympathy. }\end{array}$ \\
\hline
\end{tabular}

\section{The Expressive Techniques of the Experimental Video}

\subsection{Design Model of the Framework}

Above introducing the new video expressive technique, here is a converging model of AR and culture contents. The augmented reality technology, which was theoretically checked ahead, is a differentiated mixed reality as virtual information is added on real world. For the more, AR has a characteristic that physical devices, like mobile, are used for realizing virtual environment on the real world. Using those characteristics, I would like to propose the experimental promotional video model $\mathrm{C}$ for National Museum of Korea, which is a combination of E-museum, Virtual exhibition concepts and Augmented Reality using mobile devices [7]. The experimental model video consists of Image overlay, Information Mapping, 3D image mapping, etc. The experimental video is made using video sources taken at Nationat Museum of Korea, South Korea for the research. The experimental video is made using-Adobe Premiere pro program by adjusting Scale, Rotation, Position value of Motion effects for realizing the AR mobile applications effect, 3D image, and information mapping. The video is roughly divided in 4 scenes from scene 1 to scene 4 .

\subsection{Image Expressive Techniques}

3.2.1. Extension and Contraction using Motion Effect: The motion effects of Premiere pro are mainly used to adjust size of images or video clips by time. To adjust extension and contraction, click the image on the timeline and adjust Scale value on Effect Controls-Video 
Effects-Motion menu. Time range can be set by clicking a clock-shaped figure next to Scale value.Set point 1 on one spot by clicking the clock-shaped figure. Then adjust size of the image by changing Scale value. Set another point, point 2, on another spot in the timeline by clicking the clock-shaped figure again. Adjust Scale value for the spot, which means the previous adjusted image changes its size as time goes. Another timepoint, such as spot 3 and spot 4 , can be set up by clicking the button and the image can be adjusted on the time.By setting up the spots and values, the extension and contraction effect can be applied by time. The extension and contraction effect can be applied with image moving effect, which is used together for making Scene3, by adjusting Position value and Scale value as time processes from one point to other points. Also Opacity or Rotation value could be adjusted to present a dynamic image.

3.2.2. Image Overlay Effect: Image overlay effect makes image, video, or text stacked up on the image or video clip. This effect can be realized by adjusting Dissolve value, but the effect is applied on this experimental video by adjusting Opacity value under Motion effects section to overlap picture smoothly. Opacity value has a range of $\theta$ to 100 . By increasing the value gradually, image shows up smoothly. Also decreasing the value makes the image disappeared gradually. This effect applied on the opening and closing seene of the video, which shows the gradual change of different pictures of the museum on a smartphone image. The overlay effect makes it looks like the augmented reality image mapping, using the smartphone, which delivers the massage that user can see cloud-sourced pictures of different time or in different weather condition.

\section{Result of the Experimental Model}

\subsection{The Implement Result}

4.1.1. Scene 1: Scene 1, the opening scepe of this video, starts with text 'National Museum of Korea', a view of the miseum, and a smart phone taking pictures of it. Scene1 is designed to be seen as the smartphone scans the New and shows cloud-sourced pictures using AR applications. Pictures of different time or in different weather conditions are overlapped on the real view of the museum. By servicing AR mobile service, user sees pictures that they can't see for the moment, which broadens their experience.

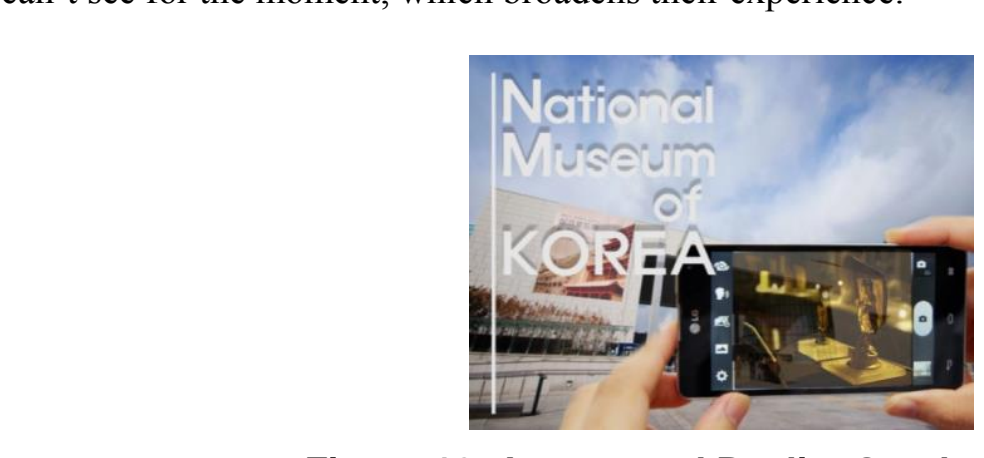

Figure 12. Augmented Reality Overlay Service

4.1.2. Scene 2: Scene 2 is about augmented reality mapping service that a smart phone scans the guide map on the wall, and AR applications detailed information pops up on the screen. The camera angle moves from left side, then stops at the first image, which is the guide map of the first floor, and 3D map made using Adobe illustrator shows up. The effect of simple schematic diagram coming up to the screen from inside of the mobile screen is 
applied by adjusting Position, Rotation, Scale, Opacity values of Motion-Effects as time processes from one point to other points. After the diagram disapears, camera angle proceeds to right side, stops at second, third guide map, and shows the schematic diagrams of each floor. The diagrams are shown as simple images in the video, however, this concept can be used as much diverse services such as showing specific information of each room or playing videos accordingly.
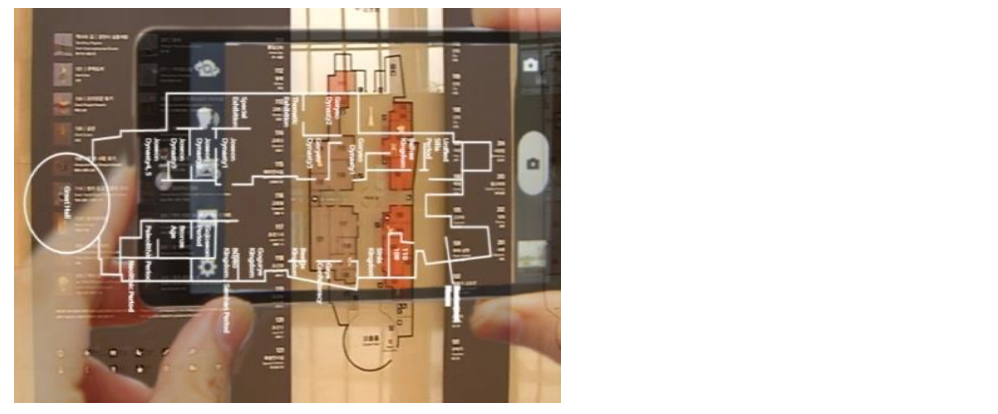

Figure 13. Presenting Guide Maps using Augmented Reality Application

4.1.3. Scene 3: Scene 3 is made using video source of Human figure, New Acquisitions of Asian Art. In the scene, the mobile device scans the human figure, and following information (Height $37.7 \mathrm{~cm}$, Human Figure etc.) shows up on the screen. This scene is based on the concept that mobile AR applications overlaps 3D moving images on actual objects and shows information of it. To express user can control size or angle of the 3D image with their fingers, Position, Rotation, Scale, and Opacity value of Motion-Effects is adjusted. This concept is useful not only for showing information and 3D images of the object, but for showing pictures of cultural assets, art pieces that do nolexist anymore. Also this concept can be used to re-call undamaged shape of the things that damaged. In other words, Augmented Reality diminishes restriction of time or space.

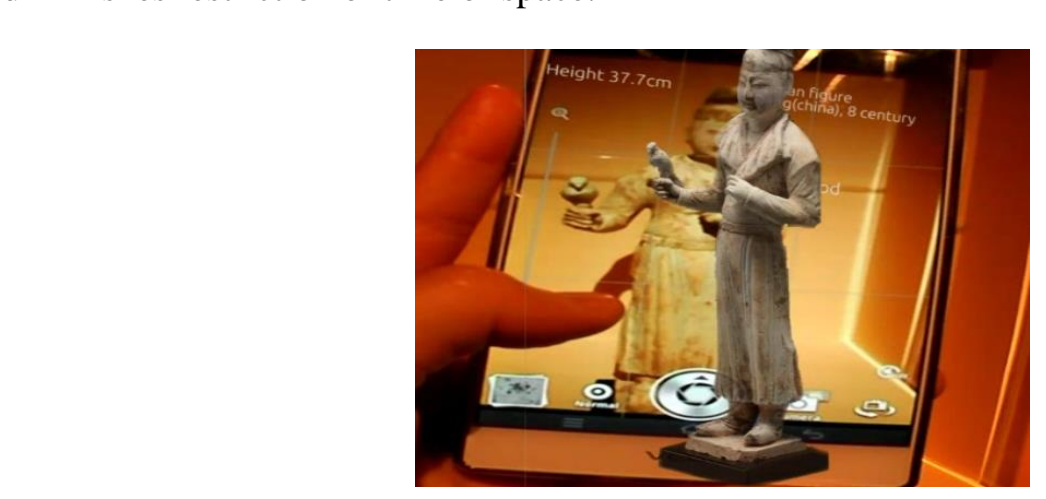

Figure 14. Image Present using Augmented Reality

4.1.4. Scene 4: As shown in Scene 2, Augmented Reality concept is usefully used to show how people interact with objects under the digital environment. The mobile device shown on the screen is a important tool for connecting the actual element, the guide map, to the virtual elements, information featured on the screen. Once the mobile scans the text 104 (means exhibition room 104), system shows detailed information of the exhibition. Specifically, information of the room, 'Buyoe Kingdom, Samhan Period' and 'Duck shaped Potery Bronze 
Tiger-shaped Buckle' shows up on the screen. Using this technique, user feels like they can easily get information of the museum and get much interested.

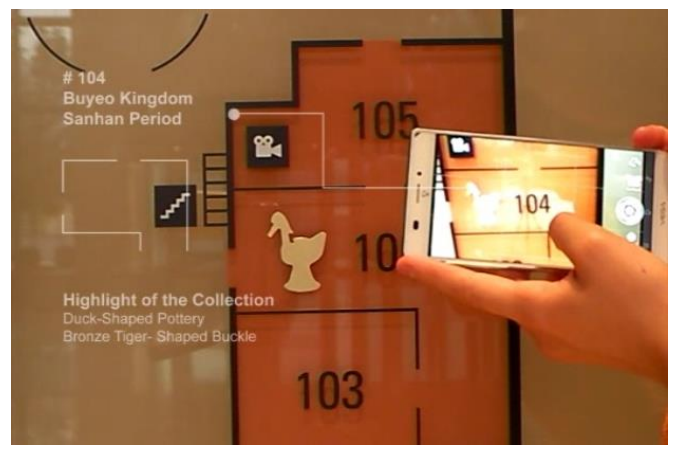

Figure 15. Guidance of Exhibition using Augmented Reality

\subsection{The Performance Evaluation Analysis}

\section{Table 3. The Performance Evaluation Analysis Table}

\begin{tabular}{|c|c|c|c|}
\hline & Promotioan video A & Promotioan video B & \\
\hline $\begin{array}{l}\text { Sample } \\
\text { Images }\end{array}$ & & & \\
\hline $\begin{array}{l}\text { Opening } \\
\text { Scene }\end{array}$ & & & \\
\hline $\begin{array}{l}\text { Ending } \\
\text { Scene }\end{array}$ & & & \\
\hline $\begin{array}{l}\text { expressive } \\
\text { technique }\end{array}$ & $\begin{array}{l}\text { Showing the location of the } \\
\text { museum using Google map } \\
\text { Delivering information } \\
\text { visuaily using video and } \\
\text { typography } \\
\text { Utilizing screen division, } \\
\text { and screen moving } \\
\text { techniques to compose } \\
\text { attractive images } \\
\text { colorful screen and lively } \\
\text { background music }\end{array}$ & $\begin{array}{l}\text { recording their Virtual } \\
\text { exhibition, realized using } \\
\text { Virtual exhibition } \\
\text { applications } \\
\text { Presenting video, 2D, and } \\
\text { 3D images together. } \\
\text { Shows how to explore the } \\
\text { virtual museum by } \\
\text { proceeding though space } \\
\text { Delivering simple } \\
\text { information with text when } \\
\text { scenes change }\end{array}$ & $\begin{array}{l}\text { Using infographic for } \\
\text { delivering information and } \\
\text { images } \\
\text { presenting dynamic AR } \\
\text { images by applying motion } \\
\text { effects } \\
\text { mixing virtual images or } \\
\text { information on the top of the } \\
\text { real world elements } \\
\text { makes people feels like they } \\
\text { are exploring the museum } \\
\text { using the mobile device and } \\
\text { the augmented reality system }\end{array}$ \\
\hline
\end{tabular}

The table above shows analyzed results of video A, B, and C. Video A is a promotional video of MUSEUM KAMPA, Prague, Czech Republic. Video B is of The Valentino Garavani Virtual Museum. Video C is National Museum of Korea, South Korea, which is the experimental video of this study. Those videos are all promotional videos of Cultural-arts 
organizations, and have a Running time of 1:30 to 2 minutes. Opening scene of video A shows the location and name of the museum using Google map and typography, and opening scene of video B consists of simple text and logo. On the other hand, Opening and ending scene of video $\mathrm{C}$ consists of interesting clips which is showing different images of outside of the museum, using a smartphone image and overlay effect. The opening and closing scenes attract viewers by giving them differentiated experience watching how the museum meets the augmented reality image mapping effect though smartphone screen. Video B is quite interesting in terms of recording their Virtual exhibition, realized using Virtual exhibition applications. However, most of scenes are virtual designed graphics which barely arouse users' emotional sympathy. On the other hand, video $\mathrm{C}$ is mixing virtual images or information on the top of the real world elements. It reproduces what people really see in the real world, and what they are hopping to do with mobile devices. Video A utilizes screen division, and screen moving techniques to compose attractive images, and video $\mathrm{B}$ presents video, 2D, and 3D images together. The scenes of video $\mathrm{C}$ are as interesting as those of video $\mathrm{A}$ or $\mathrm{B}$. video $\mathrm{C}$ shows how simple video meets augmented reality concepts and makes people feels like they are exploring the museum using the mobile device and the augmented reality system. That helps attract users' attention and enables to-make a realistic and 3-dimentinal promotional video. On the aspect of user experience and massage delivery, showing simple images and video clips could not attract users' interest and give them unconventional experience. Building an opportunity for users to interact with objects is the effective approach in the cultural-art industry and the right answer to the ICT and cultural-art converging trend. Therefore, the experience-centered massage of cultural-arts organizations can be effectively and clearly delivered to the target users thought the new expressive techniques.

\subsection{Suggestion of the New Expres 1 ye Technique Model}

Along with the new trend of uprising ICT-culure contents converging, this study proposes the new expressive techniques of cultural-an organizations' promotional video based on the research of Augmented Reality, and digitalization of culture contents such as Imaging process, or Virtual exhibition. There are growing numbers of attempt to combine ICT with cultural-art, however, it has been barety used for making promotional videos. Therefore, this study proposes the new design model for promotional video by making the experimental video of National Museun of Korea. Designing promotional videos with one of the interesting ICT techniques and E-Museum, Virtual exhibition concept enables delivering information effectively and efficiently. For the more, using mobile devices and the AR applications concept works effective with the mobile-friendly real life trend. Those techniques enable the interactive experience of consumers, expand the space by combing Virtual elements with real life, and give people a sense of reality.

\section{Concusion}

Lots of promotional videos are coming up to the market every day, and most of companies and organizations focus on promoting themselves though Web, Mobile, TV, etc. Considering this background, weak promotion strategy disappears at a moment. For this reason, this study focused on proposing the competitive promotional strategy by suggesting experimental expressive techniques using Augmented Reality, one of the uprising ICT and the digitalization, visual exhibition concept. Unlike to the existing promotional videos, which are delivering information unilaterally, the experimental video lets target users interact with culture contents using the mobile devices, and get information actively. For the more, by giving visual elements such as 3D images or Infographic, the experimental video draws user's 
attention and people get much focused on what they see. Therefore, this model video is differentiated from existing videos on the aspects of maximizing user experience and user interaction. If those techniques could be technically improved in the future, Communication between culture contents and user can be increased, and information can be delivered much more efficiently and effectively. As a result, compare to existing promotional videos are using simple video clips or images, the experimental video, which is a result of ICT-culture combined, maximizes user experience. Also the expressive techniques open up the door of ICT and cultural-art convergence.

\section{Acknowledgements}

This work was supported by a special research grant from Seoul Women's University (2014).

\section{References}

[1] J. Gomes, M. B. Carmo and A. P. Cláudio, "Creating and Assembling Virtual Exhibitions from Existing X3D Models", (2011).

[2] M. B. Carmo and A. P. Cláudio, "3D Virtual Exhibitions, DESIDOC Journal of Library and Information Technology”, vol. 33, no. 3, (2013), pp. 222-235.

[3] T. Starner, S. Mann, B. Rhodes, J. Levine, J. Healey, D. Kirsch and A. Pentland Augmented reality through wearable computing", Presence: Teleoperators and Virtual Environments, vol. 6, no. 4, (1997), pp. 386-398.

[4] R. T. Azuma, "A survey of augmented reality", Presence: Teleoperators and Virtual Environments, vol. 6, no. 4, (1997), pp. 355-385.

[5] P. Wellner, W. Mackay and R. Gold, "Computer Augmented Environments: Back to the real world", Communications of the ACM, vol. 36, no. 7,(1993), pp. 24-26

[6] P. Milgram, H. Takemura, A. Utsumi, and F. Kishino, "Atgmented reality: A class of displays on the realityvirtuality continuum", In Photonics for Industrial Applications, International Society for Optics and Photonics, (1995) December, pp. 282-292.

[7] H. Bay, B. Fasel, and L. Van Gool "Interactive museum guide: Fast and robust recognition of museum objects", (2006).

[8] S. E. Chen, "Quicktime VR:An image-based approach to virtual environment navigation", In Proceedings of the 22nd annual confererce on Computer graphics and interactive techniques, (1995) September, pp. 29-38.

[9] A. Damala, I. Marchal and P. Houlier, Merging augmented reality based features in mobile multimedia museum guides", In Proceedings of the XXI International CIPA Symposium, Athens, Greece, (2007) October, pp. 259-264.

[10] E. Rose, D. Breen, K. H. Ahers, C. Crampton, M. Tuceryan, R. Whitaker and D. Greer, "Annotating realworld objects using augnrented reality", In Computer Graphics: Developments in Virtual Environments (Proceedings of CG International'95 Conference), (1995) June, pp. 357-370.

[11] J.-s. Yun, H.-s. Kang and I.-y. Moon, "Analysis study of movement patterns using BigData analysis technology", Journal of the Korea Institute of Information and Communication Engineering, vol. 18, no. 5, (2014) May, pp. 1073-1079.

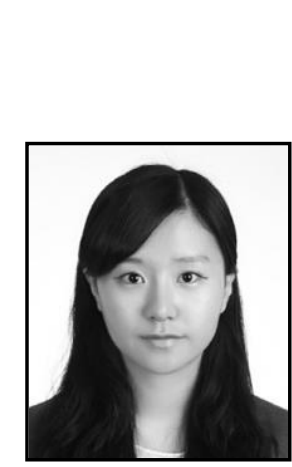

\section{Authors}

\section{Ga Ram Choi}

Seoul Women's University

Department of Contents Design

Digital Contents Lab

Researcher

grchoi@dclab.kr 


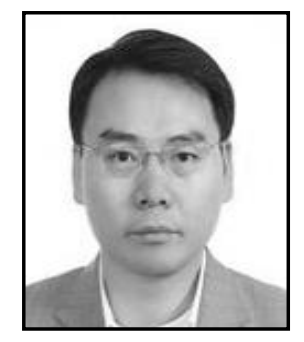

HaK Hyun Choi (Corresponding author)

Korea University, Ph.D. Course

Seoul Women's University, Professor

Department of Contents Design

Digital Contents Lab

choiidea@gmail.com

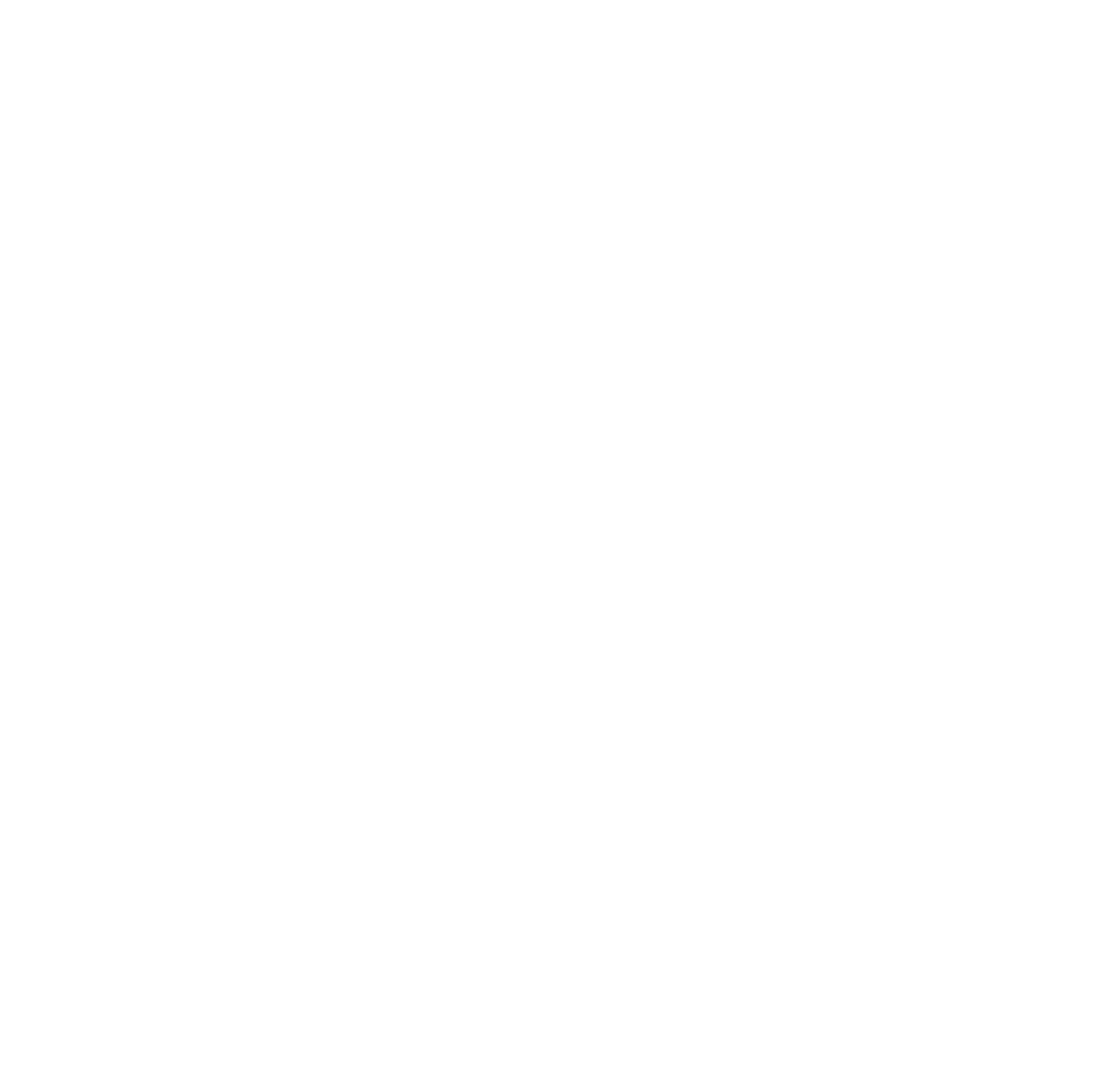


International Journal of Smart Home

Vol.8, No.5 (2014)

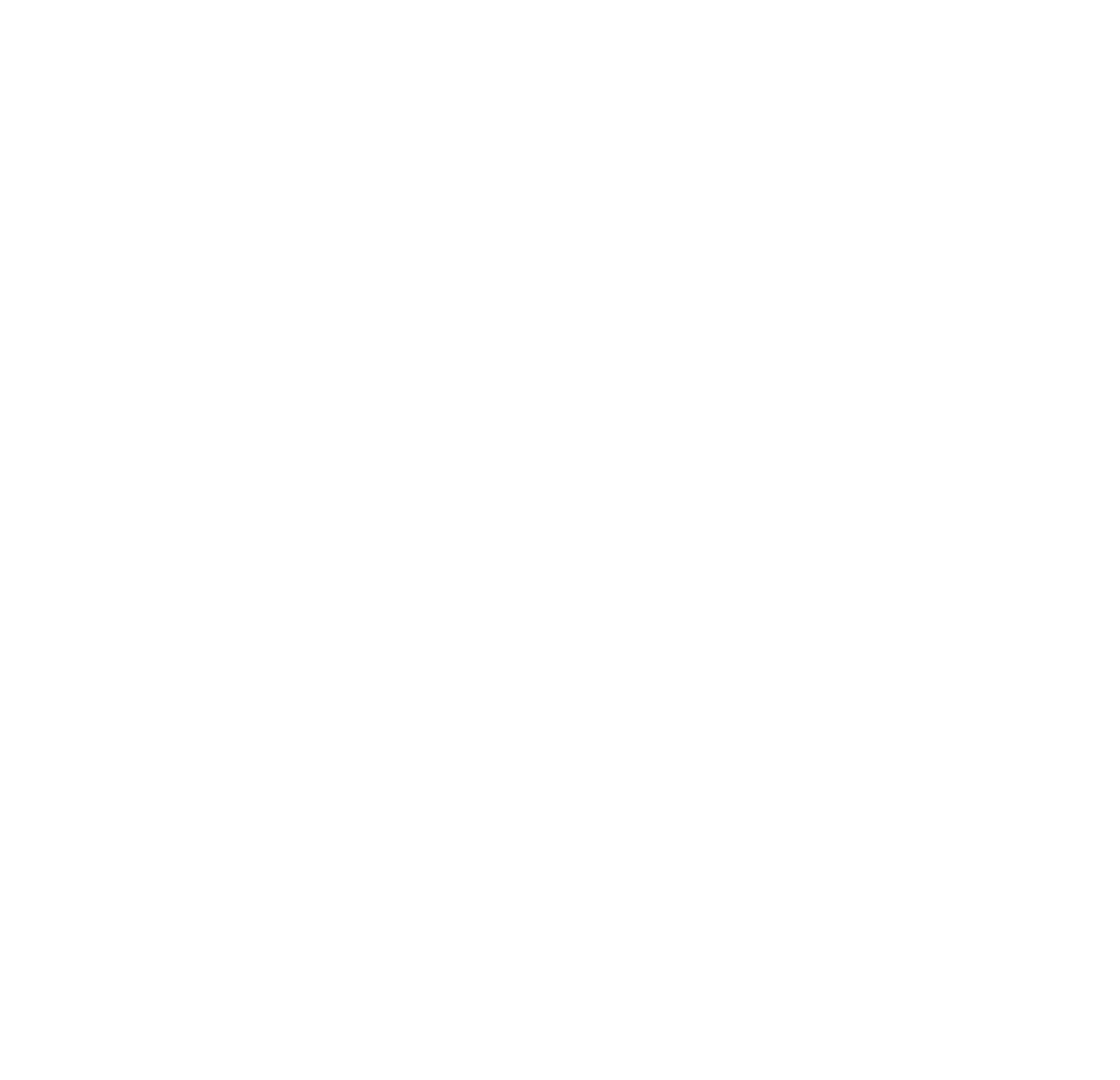

